\title{
MULTIPLICATIVE FUNCTIONALS ON SEMIGROUPS OF CONTINUOUS FUNCTIONS
}

\section{H. S. BEAR AND BERTRAM YOOD ${ }^{1}$}

Let $X$ be a compact Hausdorff space. We denote by $C(X)$ the multiplicative semigroup of all continuous real-valued functions on $X$. Milgram [2] has shown that $C(X)$ as a semigroup determines $X$. In this paper we investigate the set $\mathfrak{F}(X)$ of all continuous positive nontrivial ${ }^{2}$ multiplicative functionals on $C(X)$, where $C(X)$ has the topology of uniform convergence. If multiplication is defined pointwise, $(F \cdot G)(f)=F(f) \cdot G(f)$ for $F, G \in \mathfrak{F}(X), f \in C(X)$ then $\mathfrak{F}(X)$ as a semigroup determines the space $X$ for spaces satisfying the first axiom of countability, but not, in general, otherwise. We find the general form of semigroup isomorphisms of $\mathfrak{F}\left(X_{1}\right)$ onto $\mathfrak{F}\left(X_{2}\right)$ which are "continuous" in a suitable sense in the case where the spaces satisfy the first axiom of countability.

Bourgin [1] and Turowicz [3] have shown that if $F \in \mathfrak{F}(X)$, there is a uniquely determined countable closed set $\left\{x_{n}\right\}$ and a summable sequence $\left\{a_{i}\right\}$ of positive numbers such that $F(f)=\prod\left|f\left(x_{i}\right)\right|^{a_{i}}$. Conversely, any functional $F$ defined in this way is continuous. To obtain this representation of the multiplicative functional $F$, one represents as an integral the corresponding linear functional $L$ defined by $L(f)$ $=\log F\left(e^{f}\right)$. Thus $L(f)=\int f d \mu$, and $F(g)=\exp \int \log g d \mu$, for positive functions $g$. One can then prove that $\mu$ is a positive measure whose support is a countable closed set $\left\{x_{i}\right\}$, and the representation for $F$ follows. We denote by $D(F)$ the countable closed set $\left\{x_{n}\right\}$ which occurs in the representation of $F$.

If $S(X)$ is the semigroup of all continuous multiplicative functionals on $C(X)$, then $\mathfrak{F}(X)$ can be algebraically characterized in $S(X)$ as the set of functionals which are squares. Therefore if $S\left(X_{1}\right)$ is isomorphic to $S\left(X_{2}\right)$, it follows that $\mathfrak{F}\left(X_{1}\right)$ is isomorphic to $\mathfrak{F}\left(X_{2}\right)$ and our results for $\mathfrak{F}(X)$ imply analogous results for $S(X)$.

By an ideal in $\mathfrak{F}(X)$ we mean a subset $I$ with the property that $F \in I, G \in \mathfrak{F}(X)$ imply that $F G \in I$. An ideal $I$ is called a $P$-ideal if for each pair of elements $F_{1}, F_{2}$ in $I$ there exist $G \in I$, and $H_{1}, H_{2}$ in

Presented to the Society, August 24, 1956; received by the editors December 16, 1958.

1 The work of this author was supported in part by the National Science Foundation, Research Grant NSF-G2573.

2 That is, $F(f) \geq 0$ for all $f$ in $C(X)$ and $F$ is neither identically zero nor identically one. 
$\mathfrak{F}(X)$ such that $F_{i}=G H_{i}, i=1,2$. By a simple application of Zorn's maximum principle we obtain

Lemma 1. Every $P$-ideal is contained in a maximal $P$-ideal.

Lemma 2. If $I$ is a $P$-ideal, $F_{1}, \cdots, F_{n} \in I$ then there exist $G \in I$, $H_{1}, \cdots, H_{n} \in \mathfrak{F}(X)$ such that $F_{i}=G H_{i}, i=1, \cdots, n$.

Proof. We proceed by induction. Suppose the conclusion holds for $n$ elements and let $F_{1}, \cdots, F_{n+1} \in I$ where $F_{i}=G H_{i}, i=1, \cdots, n$, $G \in I$. There exist $G_{1} \in I, K_{1}, K_{2} \in \mathfrak{F}(X)$ such that $F_{n+1}=G_{1} K_{1}$, $G=G_{1} K_{2}$. Then $F_{1}, \cdots, F_{n+1}$ are multiples of $G_{1} \in I$.

For $x \in X$ let $I_{x}=\{F \in \mathfrak{F}(X): x \in D(F)\}$.

Lemma 3. For each $x \in X, I_{x}$ is a maximal $P$-ideal of $\mathfrak{F}(X)$. All maximal $P$-ideals of $\mathfrak{F}(X)$ have this form.

Proof. Let $I$ be a $P$-ideal, and $F_{1}, \cdots, F_{n} \in I$. Then Lemma 2 shows that the intersection of the sets $D\left(F_{1}\right), \cdots, D\left(F_{n}\right)$ is not void since it contains $D(G)$ where $G$ is a common factor in $I$ of $F_{1}, \cdots, F_{n}$. Since $X$ is compact, $\cap\{D(F): F \in I\}$ is nonvoid. For any $x$ in this set, $I \subset I_{x}$. We show next that $I_{x}$ is a $P$-ideal. For let $F_{1}, F_{2} \in I_{x}$. We set $F_{1}(f)=|f(x)|^{p_{1}} \prod\left|f\left(t_{i}\right)\right|^{a_{i}}$ and $F_{2}(f)=|f(x)|^{p_{2}} \prod\left|f\left(u_{i}\right)\right|^{b_{i}}$. Let $p_{1}$ $=p+q_{1}, p_{2}=p+q_{2}$ where $p, q_{1}$ and $q_{2}$ are positive. Then $F_{1}$ and $F_{2}$ have $G \in I_{x}$ as a common factor where $G$ is defined by $G(f)=|f(x)|^{p}$.

To show that $I_{x}$ is a maximal $P$-ideal assume on the contrary that $I_{x}$ is properly contained in a $P$-ideal $I$. Let $F_{1} \in I, F_{1} \in I_{x}$ and let $F_{2}$ be defined by $F_{2}(f)=|f(x)|$. Then $x \notin D\left(F_{1}\right)$ and $D\left(F_{1}\right) \cap D\left(F_{2}\right)=0$ which is impossible. The second statement is clear.

It is readily seen that if $x \neq y$ in $X$ then $I_{x} \neq I_{y}$. The mapping $x \rightarrow I_{x}$ is a one-to-one mapping of $X$ onto the collection of all maximal $P$-ideals of $\mathfrak{F}(X)$.

Theorem 1. Let $X_{1}, X_{2}$ be two compact Hausdorff spaces. Then the following statements are equivalent.

(1) There is a semigroup isomorphism $\tau$ of $\mathfrak{F}\left(X_{1}\right)$ onto $\mathfrak{F}\left(X_{2}\right)$.

(2) There is a semigroup homomorphism $\sigma$ of $\mathfrak{F}\left(X_{1}\right)$ onto $\mathfrak{F}\left(X_{2}\right)$ such that $D\left(F_{1}\right)=D\left(F_{2}\right)$ whenever $\sigma\left(F_{1}\right)=\sigma\left(F_{2}\right)$.

(3) There is a semigroup homomorphism $\sigma$ of $\mathfrak{F}\left(X_{1}\right)$ onto $\mathfrak{F}\left(X_{2}\right)$ such that, for any maximal $P$-ideal $N$ of $\mathfrak{F}\left(X_{2}\right), \sigma^{-1}(N)$ is contained in a $P$-ideal of $\mathfrak{F}\left(X_{1}\right)$.

(4) There is a 1-1 mapping $\psi$ of $X_{1}$ onto $X_{2}$ such that for each countable set $Q$ in $X_{1}, \psi[Q]$ is closed if and only if $Q$ is closed.

Proof. Clearly (1) implies (2). Suppose (2) holds. We show that the mapping $\sigma$ of (2) has the property of (3). Let $N$ be a maximal $P$ - 
ideal of $\mathfrak{F}\left(X_{2}\right)$. Let $F_{1}, \cdots, F_{n} \in \sigma^{-1}(N)$. By Lemma 2 there exist $\sigma(G) \in N, \sigma\left(H_{i}\right) \in \mathfrak{F}\left(X_{2}\right), i=1, \cdots, n$ such that $\sigma\left(F_{i}\right)=\sigma(G) \sigma\left(H_{i}\right)$ $=\sigma\left(G H_{i}\right)$ and therefore, by (2), $D\left(F_{i}\right)=D\left(G H_{i}\right) \supset D(G)$ for each $i$. Hence $\cap\left\{D(F): F \in \sigma^{-1}(N)\right\} \neq 0$. For $x$ in this intersection, $\sigma^{-1}(N)$ $\subset I_{x}$.

Assume (3). Let $P_{1}, P_{2}$ be the collections of all maximal $P$-ideals of $\mathfrak{F}\left(X_{1}\right), \mathfrak{F}\left(X_{2}\right)$, respectively. We first show that if $M \in P_{1}$, then $\sigma[M] \in P_{2}$, and that if $N \in P_{2}$, then $\sigma^{-1}[N] \in P_{1}$. Note that $\sigma[M]$ is a $P$-ideal of $\mathfrak{F}\left(X_{2}\right)$. By Lemma 1 there exists $N_{1} \in P_{2}$ such that $\sigma[M] \subset N_{1}$, and hence $M \subset \sigma^{-1}\left[N_{1}\right] \subset M_{1}$ for some $M_{1} \in P_{1}$. It follows that $M=\sigma^{-1}\left[N_{1}\right]$, and $\sigma[M]=N_{1}$ since $M$ is maximal. If $N \in P_{2}$, then, by hypothesis, $\sigma^{-1}[N] \subset M_{1}$ for some $M_{1} \in P_{1}$. Therefore $N \subset \sigma\left[M_{1}\right]$. But we have just seen that $\sigma\left[M_{1}\right] \in P_{2}$, so $N=\sigma\left[M_{1}\right]$ and $\sigma^{-1}[N]$ $=M_{1}$. Thus $\sigma$ can be considered as a 1-1 mapping of $P_{1}$ onto $P_{2}$. We have $\sigma^{-1} \sigma[M]=M$ and $F \in M$ if and only if $\sigma(F) \in \sigma[M]$ for all $M \in P_{1}$.

The above shows that for any $x \in X_{1}$ there is a unique $y \in X_{2}$ such that $\sigma\left[I_{x}\right]=I_{\boldsymbol{y}}$. We define $\psi(x)=y$, and show that $\psi$ is the one-to-one mapping in (4). For any countable closed set $H$ in $X_{1}$, there is $F \in \mathfrak{F}\left(X_{1}\right)$ such that $D(F)=H$. Since $F \in I_{x}$ is equivalent to $x \in D(F)$ and also to $\sigma(F) \in I_{\psi(x)}$, we have $\psi(x) \in D(\sigma(F))$ if and only if $x \in H$ $=D(F)$. Thus $\psi[H]$ is closed in $X_{2}$. Similarly, if $K$ is a countable closed set in $X_{2}$, let $G=\sigma(F)$ and $D(G)=K$. Then $\psi[D(F)]=K$, and $D(F)$ is necessarily closed.

Assume (4). Since the functionals in $\mathfrak{F}\left(X_{1}\right)$ and $\mathfrak{F}\left(X_{2}\right)$ are determined by closed sequences in $X_{1}$ and $X_{2}$ and sequences of numbers, it is clear that the function $\psi$ induces a semigroup isomorphism $\tau$ of $\mathfrak{F}\left(X_{1}\right)$ onto $\mathfrak{F}\left(X_{2}\right)$. This shows that (4) implies (1) and completes the proof of the theorem.

Corollary. If $X_{1}, X_{2}$ are compact Hausdorff spaces satisfying the first axiom of countability, then $X_{1}$ is homeomorphic to $X_{2}$ if and only if $\mathfrak{F}\left(X_{1}\right)$ is isomorphic to $\mathfrak{F}\left(X_{2}\right)$.

Proof. In a space satisfying the first axiom of countability, the countable closed sets determine the topology, and hence the function $\psi$ is a homeomorphism.

It is possible to have $\mathfrak{F}\left(X_{1}\right)$ isomorphic to $\mathfrak{F}\left(X_{2}\right)$ and $X_{1}$ not homeomorphic to $X_{2}$ since there are spaces which are compact and Hausdorff under two different topologies which have the same countable closed sets.

Lemma 4. A homomorphism $\sigma$ of $F\left(X_{1}\right)$ onto $F\left(X_{2}\right)$ satisfies (2) of Theorem 1 if and only if it satisfies (3) of Theorem 1. 
Proof. As shown above, if $\sigma$ satisfies (2) then $\sigma$ satisfies (3). But if $\sigma$ satisfies (3), the proof of Theorem 1 shows that $D(F)=\psi^{-1} D(\sigma(F))$ so that (2) holds.

Let $\sigma$ be a homomorphism of $\mathfrak{F}\left(X_{1}\right)$ onto $\mathfrak{F}\left(X_{2}\right)$. We call $\sigma$ sequentially continuous if $F_{n}(f) \rightarrow F(f)$ for all $f \in C\left(X_{1}\right)$ implies $\sigma\left(F_{n}\right)(g)$ $\rightarrow \sigma(F)(g)$ for all $g \in C\left(X_{2}\right)$.

THEOREM 2. Let $\sigma$ be a sequentially continuous homomorphism of $\mathfrak{F}\left(X_{1}\right)$ onto $\mathfrak{F}\left(X_{2}\right)$. If $\sigma$ satisfies (2) or (3) of Theorem 1 , then $\sigma$ is an isomorphism.

Proof. Consider all $F \in \mathfrak{F}\left(X_{1}\right)$ which lie in the maximal $P$-ideal $I_{t}$ and are in no other maximal $P$-ideal. These are the functionals $F$ of the form $F(f)=|f(t)|^{\alpha}$. Then $\sigma(F)$ has the form $\sigma(F)(g)$ $=|g(\psi(t))|^{\beta(\alpha)}$. Since $\sigma$ is a homomorphism, $\beta(\alpha+\gamma)=\beta(\alpha)+\beta(\gamma)$ for $\alpha>0, \gamma>0$. We show that $\beta(\alpha)$ is continuous. Suppose $\alpha_{n} \rightarrow \alpha_{0}>0$. Set $F_{n}(f)=|f(t)|^{\alpha_{n}}$. Then $F_{n}(f) \rightarrow|f(t)|^{\alpha_{0}}$. Consequently $\sigma\left(F_{n}\right)(g)$ $=|g(\psi(t))|^{\beta\left(\alpha_{n}\right)} \rightarrow|g(\psi(t))|^{\beta\left(\alpha_{0}\right)}$. By taking $g$ identically two we see that $\beta\left(\alpha_{n}\right) \rightarrow \beta\left(\alpha_{0}\right)$. Therefore there is a number $c(t)>0$ such that $\beta(\alpha)=c(t) \alpha$. Let

$$
F(f)=\prod_{k=1}^{\infty}\left|f\left(t_{k}\right)\right|^{a_{k}}=\lim _{n} F_{n}(f)
$$

where

$$
F_{n}(f)=\prod_{k=1}^{n}\left|f\left(t_{k}\right)\right|^{a_{k}} .
$$

Then $\sigma(F)(g)=\lim \sigma\left(F_{n}\right)(g)$ whence

$$
\sigma(F)(g)=\prod_{k=1}^{\infty}\left|g\left(\psi\left(t_{k}\right)\right)\right| c\left(t_{k}\right) a_{k} .
$$

Suppose that $\sigma\left(F_{1}\right)=\sigma(F)$. Then $D\left(F_{1}\right)=D(F)$ by hypothesis (and Lemma 4). Thus we may write

$$
F_{1}(f)=\prod_{k=1}^{\infty}\left|f\left(t_{k}\right)\right|^{b_{k}}
$$

and hence

$$
\sigma(F)(g)=\sigma\left(F_{1}\right)(g)=\prod_{k=1}^{\infty}\left|g\left(\psi\left(t_{k}\right)\right)\right|^{e\left(t_{k}\right) b_{k}} .
$$

It follows that $c\left(t_{k}\right) a_{k}=c\left(t_{k}\right) b_{k}$ or $a_{k}=b_{k}$ for each $k$. Therefore $F=F_{1}$. 
Theorem 3. Let $X_{1}, X_{2}$ be two compact Hausdorff spaces satisfying the first axiom of countability. Let $\sigma$ be a sequentially continuous isomorphism of $\mathfrak{F}\left(X_{1}\right)$ onto $\mathfrak{F}\left(X_{2}\right)$. Then there exists a continuous semigroup isomorphism s of $C\left(X_{2}\right)$ onto $C\left(X_{1}\right)$ such that $\sigma(F)(g)=F(s(g))$ for all $F \in \mathfrak{F}\left(X_{1}\right), g \in C\left(X_{2}\right)$. Conversely any $\sigma$ of this form is sequentially continuous.

Proof. By Corollary 1 the mapping $\psi$ of Theorem 1 is a homeomorphism of $X_{1}$ onto $X_{2}$. As shown in the proof of Theorem 2, there exists a positive function $c(t)$ defined on $X_{1}$ such that if $F \in \mathfrak{F}\left(X_{1}\right)$ is given by

$$
F(f)=\prod_{k=1}^{\infty}\left|f\left(t_{k}\right)\right|^{a_{k}} . \quad \quad f \in C\left(X_{1}\right)
$$

then

$$
\sigma(F)(g)=\prod_{k=1}^{\infty}\left|g\left(\psi\left(l_{k}\right)\right)\right| a_{k} c\left(t_{k}\right) \quad g \in C\left(X_{2}\right) .
$$

We show that $c(t)$ is continuous. Let $t_{n} \rightarrow t_{0}$ in $X_{1}$ and let $F_{n}(f)$ $=\left|f\left(t_{n}\right)\right|$ and $F(f)=\left|f\left(t_{0}\right)\right|$. Then $F_{n}(f) \rightarrow F(f)$ and consequently $\sigma\left(F_{n}\right)(g) \rightarrow \sigma(F)(g)$. But then $\left|g\left(\psi\left(t_{n}\right)\right)\right|^{c\left(t_{n}\right)} \rightarrow\left|g\left(\psi\left(t_{0}\right)\right)\right| c\left(t_{0}\right)$. By taking $g$ identically two we see that $c\left(t_{n}\right) \rightarrow c\left(t_{0}\right)$.

Set $p(u)=c\left(\psi^{-1}(u)\right), u \in X_{2}$. Then $p(\psi(t))=c(t), t \in X_{1}$. We then obtain

$$
\begin{aligned}
\sigma(F)(g) & =\prod_{k=1}^{\infty}\left|g\left(\psi\left(t_{k}\right)\right)\right|^{a_{k} p\left(\psi\left(t_{k}\right)\right)}=\prod_{k=1}^{\infty}\left\{\left|g\left(\psi\left(t_{k}\right)\right)\right| p\left(\psi\left(t_{k}\right)\right)\right\} a^{a_{k}} \\
& =F\left(|g(\psi(\cdot))|^{p(\psi(\cdot))}\right) .
\end{aligned}
$$

Now define a mapping $s(g)$ of $C\left(X_{2}\right)$ into $C\left(X_{1}\right)$ by the rule $s(g)(t)$ $=\operatorname{sgn}[g(\psi(t))]|g(\psi(t))|^{p(\psi(t))}$. Recall that $p(\psi(t))>0$ and is continuous. A routine argument shows that $s$ is a continuous semigroup isomorphism of $C\left(X_{2}\right)$ onto $C\left(X_{1}\right)$. We need also the fact that $F(h)$ $=F(|h|)$ for $F \in \mathfrak{F}\left(X_{1}\right)$. Therefore $\sigma(F)(g)=F(|s(g)|)=F(s(g))$. The converse is clear.

The above proof also shows that any continuous isomorphism $s$ of $C\left(X_{2}\right)$ onto $C\left(X_{1}\right)$ has the form $|s(g)(t)|=|g(\psi(t))|^{p(\psi(t))}$ where $\psi$ is a homeomorphism of $X_{1}$ onto $X_{2}$ and $p$ is a continuous positive function on $X_{1}$. For, given the isomorphism $s$, define $\sigma$ by $\sigma(F)(g)=F(s(g))$. Then $\sigma$ is sequentially continuous and the formulas of Theorem 3 give

$$
F(|s(g)|)=F(s(g))=F(|g(\psi(\cdot))| p(\psi(\cdot)))
$$


for suitable $\psi$ and $p$. Since $F$ is arbitrary in $\mathfrak{F}\left(X_{1}\right)$, the relation for $s(g)(t)$ follows. This conclusion can also be deduced from a theorem of Milgram [2, p. 383].

\section{REFERENCES}

1. D. G. Bourgin, Multiplicative transformations, Proc. Nat. Acad. Sci. U.S.A. vol. 36 (1950) pp. 564-570.

2. A. N. Milgram, Multiplicative semigroups of continuous functions, Duke Math. J. vol. 16 (1949) pp. 377-383.

3. A. Turowicz, Sur les fonctionelles continues et multiplicatives, Ann. Soc. Polon. Math. vol. 20 (1947) pp. 135-156.

\section{UNIVERSITY OF WASHINGTON AND \\ UNIVERSITY OF OREGON}

\section{SIMULTANEOUS APPROXIMATION BY A POLYNOMIAL AND ITS DERIVATIVES}

\section{ERRETT BISHOP ${ }^{1}$}

1. Introduction. We shall prove the following theorem:

THEOREM. If $f_{0}$ is a continuous function on a compact set $C$ without interior and with connected complement of the complex plane, and if $f_{1}, \cdots, f_{n}$ are continuous functions on a compact totally disconnected subset $E$ of $C$, then there exists a sequence $\left\{p_{i}\right\}$ of polynomials such that $p_{i} \rightarrow f_{0}$ uniformly on $C$ as $i \rightarrow \infty$, and $p_{i}{ }^{[k]} \rightarrow f_{k}$ uniformly on $E$ as $i \rightarrow \infty$, for $1 \leqq k \leqq n$, where $p_{i}{ }^{[k]}$ is the kth derivative of $p_{i}$.

For the case in which $E$ is void, this reduces to a well-known theorem of Lavrentiev [3]. A different case of the problem of simultaneous approximation by a polynomial and its derivatives-the problem of simultaneous approximation on a Jordan arc-has been considered in a previous paper [1], by methods distinct from the methods of the present paper.

We make some preliminary remarks concerning distributions. The distributions in question will all be defined on the complex plane and have complex values and have a compact support. If $T$ is such a distribution and if $f$ is a function defined on the complex plane and having partial derivatives of all orders, then $\langle f, T\rangle$ will denote the

Received by the editors March 26, 1958 and, in revised form, December 1, 1958.

1 This work was partially supported by the Office of Naval Research through Contract ONR 041157. 